\title{
The Utility of Rapid On-Site Evaluation on Endobronchial Ultrasound Guided Transbronchial Needle Aspiration: Does It Make a Difference?
}

\author{
Raymond W. M. Wong, ${ }^{1}$ Alesha Thai, ${ }^{1}$ Yet H. Khor, ${ }^{1}$ Kerryn Ireland-Jenkin, ${ }^{2}$ \\ Celia J. Lanteri, ${ }^{1}$ and Barton R. Jennings ${ }^{1}$ \\ ${ }^{1}$ Department of Respiratory and Sleep Medicine, Austin Health, Melbourne, VIC 3084, Australia \\ ${ }^{2}$ Department of Pathology, Austin Health, Melbourne, VIC 3084, Australia
}

Correspondence should be addressed to Barton R. Jennings; bjennings@lungandsleep.com.au

Received 30 August 2014; Revised 22 October 2014; Accepted 24 October 2014; Published 12 November 2014

Academic Editor: Takeshi Mori

Copyright (C) 2014 Raymond W. M. Wong et al. This is an open access article distributed under the Creative Commons Attribution License, which permits unrestricted use, distribution, and reproduction in any medium, provided the original work is properly cited.

\begin{abstract}
The purpose of this study was to assess the efficacy of using rapid on-site evaluation (ROSE) for samples taken during endobronchial ultrasound guided transbronchial needle aspiration (EBUS-TBNA) at Austin Health, Victoria. This was compared to data collected for cases performed without ROSE. A retrospective analysis was conducted on 188 consecutive patients who underwent EBUSTBNA from May 2012 to July 2014 whose data was collected prospectively at the time of the procedure. The presence of a cytologist during ROSE resulted in a significant reduction in the number of lesions sampled [mean: $1.5 \pm 0.7(1,4)$ versus $1.9 \pm 0.8(1,4), P=$ 0.0020 ] and the number of TBNAs required per case [mean: $3.6 \pm 1.4(1,8)$ versus $4.2 \pm 1.5(1,8), P=0.0017$ ]. This could potentially result in a shorter procedure time and, ultimately, a reduction in complication rate. The quality of the samples obtained during EBUS-TBNA with ROSE was higher. A larger proportion of samples yielded a satisfactory cell block allowing the potential benefit of additional pathology testing including immunohistochemistry and molecular pathology. In summary, the use of ROSE during EBUS-TBNA was superior to off-site cytological assessment of bronchoscopy specimens.
\end{abstract}

\section{Introduction}

Endobronchial ultrasound guided transbronchial needle aspiration (EBUS-TBNA) is widely used for tissue sampling of mediastinal and hilar lesions adjacent to the proximal airway. Indications for this procedure include mediastinal lymph node staging in patients with non-small cell lung cancer, assessment of mediastinal or hilar lymphadenopathy, and direct sampling of lung lesions adjacent to the proximal airway.

EBUS increases the diagnostic yield of blind TBNA and reduces complications by allowing real-time ultrasound guidance during needle insertion $[1,2]$. EBUS also allows for a less invasive approach to various lymph node stations in the mediastinum and hilum compared to video-assisted thoracoscopic surgery (VATS) or mediastinoscopy [3].
Rapid on-site evaluation (ROSE) is a technique where TBNA cytology samples are rapidly stained and screened for diagnostic material in the procedure room, during the procedure. There is a high agreement between the on-site and final pathologic evaluation of EBUS-TBNA specimens [4].

The use of ROSE in conjunction with EBUS-TBNA has been shown to improve the diagnostic yield of the procedure [5-8]. ROSE also significantly improved the number of specimens that were adequate for a diagnosis of central lung cancers adjacent to the airway or lymph node metastases as described by Davenport [8]. Baram et al. demonstrated that when ROSE confirmed diagnostic material, this frequently spared the need for additional sampling and the overall cost of the procedure was lowered [4]. This can prevent a repeat bronchoscopy or other invasive surgical procedures, which may lead to complication. 
ROSE provides an opportunity to adjust sampling sites during EBUS-TBNA based on the results obtained. If the cytologist declares that diagnostic material has been harvested in sufficient quantity and quality the procedure can be finished leading to reduced procedure time. If there is no diagnostic tissue seen, the bronchoscopist can sample the same site again or go on and sample the next site. This can allow TBNA samples to be more efficiently targeted to improve diagnostic yield and reduce procedure time. This study did not assess procedure time.

Prior to the implementation of ROSE at Austin Health, EBUS-TBNA samples were smeared on a slide and fixed in the procedure room. The slides were then taken to the cytology department within the same hospital and analysed by a cytologist. Preliminary results were conveyed to the bronchoscopist via telephone. Taking the slides to the cytology department for analysis led to a significant delay before the bronchoscopist got the result. ROSE has the benefit of avoiding this delay and, therefore, having greater impact on the procedure.

We hypothesised that the implementation of ROSE during EBUS-TBNA will reduce the number of lymph node aspirates performed during bronchoscopy, with no reduction in the quality and diagnostic utility of the specimen achieved.

\section{Patients and Methods}

A prospective database for interventional bronchoscopies was developed at Austin Health in May 2012. All patients undergoing EBUS-TBNA at the Department of Respiratory and Sleep Medicine for the evaluation of mediastinal or hilar lesions between May 2012 and July 2014 were retrospectively analysed.

The number of lymph node stations sampled, number of aspirations per lymph node station, total number of aspirations made per case, and number of patients who had more than one lesion sampled were recorded.

Prospective data was collected for all patients undergoing EBUS-TBNA from 4 June 2013 after the implementation of ROSE and was compared to the same data which was retrospectively collected for 69 patients who underwent linear EBUS prior to the implementation of ROSE (from May 2012 to April 2013).

The study was approved by the ethical committee of Austin Health, Victoria. This study was a retrospective review of results and was classified as a Low and Negligible Risk Research (LNRR) Project (Project number: LNR/13/Austin/ 216), and hence, informed consent was not necessary.

2.1. EBUS-TBNA. The procedure was performed using an integrated fibreoptic bronchoscope (BF-UC160F-OL8) as previously described $[9,10]$, with a dedicated $22-\mathrm{G}$ TBNA needle, by either an experienced bronchoscopist or a supervised advanced trainee in respiratory medicine.

Sites for TBNA were chosen based on clinical tumour stage, computed tomography scan, and intraprocedural findings. EBUS-TBNA was performed under conscious sedation with midazolam and fentanyl given by the bronchoscopist.
Local anaesthesia was achieved with lignocaine spray into the pharynx immediately prior to the procedure. Additional $2 \%$ lignocaine boluses were also used as required throughout the procedure. Patients were monitored with continuous cardiac monitoring including blood pressure, as well as pulse oximetry, without an anaesthetist.

2.2. Rapid On-Site Evaluation of Specimens. During bronchoscopy, material obtained from transbronchial needle aspiration was transferred onto numbered glass slides. At least one air-dried smear and one 95\% alcohol fixed smear were prepared from each aspiration. The remainder of the aspirated material was put into a saline pot to create a cell block. As part of ROSE, the cytologist assessed the adequacy of the sample, defined by the presence and number of lymphocytes and whether there was diagnostic material present after each aspiration. The bronchoscopist decided when to stop performing TBNAs based on result of ROSE and patient tolerance of the procedure. Prior to ROSE, the procedure was ended when adequate number of TBNA samples were performed to maximize the chance of a useful result. The plan as to which lymph node stations to sample was made prior to the procedure based on the CT and PET findings. Usually three TBNA samples were performed per station but this would be adjusted depending on patient tolerance of the procedure. Usually this would be achieved before the cytologist conveyed the result of the first TBNA by phone. If the procedure was prolonged and the cytologist confirmed a positive and adequate sample, the procedure was ceased at that time.

2.3. Statistical Analysis. Statistical analysis was performed using Graphpad Prism (v5, Graphpad Software, USA). Data distributions were tested for normality using the Kolmogorov-Smirnov test. Parametric distributions were analysed with $U$-tests for comparisons of the two groups. Correlations were assessed using Pearson's test. For nonparametric data, Mann-Whitney test was used for the two-group comparison. Nonparametric correlations were assessed using Spearman's test.

\section{Results}

Between May 2012 and July 2014, 188 consecutive patients were enrolled in this study. Seventy-one patients underwent EBUS prior to the implementation of ROSE. Four patients were excluded as no TBNA samples were taken. In the post-ROSE group, 117 patients who underwent EBUS were included. Five patients were excluded, as TBNA was not performed. Thus, a total of 179 patients (66 patients in preROSE group, 112 patients in post-ROSE group) were included in this study. Baseline characteristics of the patients are shown in Table 1.

The primary diagnoses were as follows: small cell carcinoma (29), adenocarcinoma (26), non-small cell lung cancer (not otherwise specified) (12), squamous cell carcinoma (3), granulomas (45), and others (14) which include metastatic melanoma, neuroendocrine tumour, metastatic breast cancer, 
TABLE 1: Baseline patient characteristics.

\begin{tabular}{lcc}
\hline Characteristics & Pre-ROSE & Post-ROSE \\
\hline Patients & 66 & 112 \\
Gender (M/F) & $44 / 27$ & $75 / 42$ \\
Age, years (mean) & 60.4 & 59.5 \\
Target lesions (\%) & & \\
2R & 0 & 3 \\
2L & 0 & 0 \\
3p & 0 & 4 \\
$4 \mathrm{R}$ & 49 & 28 \\
$4 \mathrm{~L}$ & 9 & 11 \\
7 & 60 & 54 \\
$10 \mathrm{R}$ & 3 & 3 \\
$10 \mathrm{~L}$ & 3 & 0 \\
11R & 33 & 21 \\
11L & 19 & 10 \\
$12 \mathrm{R}$ & 0 & 0 \\
Lung mass & 18 & 19 \\
\hline
\end{tabular}

Data presented as $n$ unless specified.

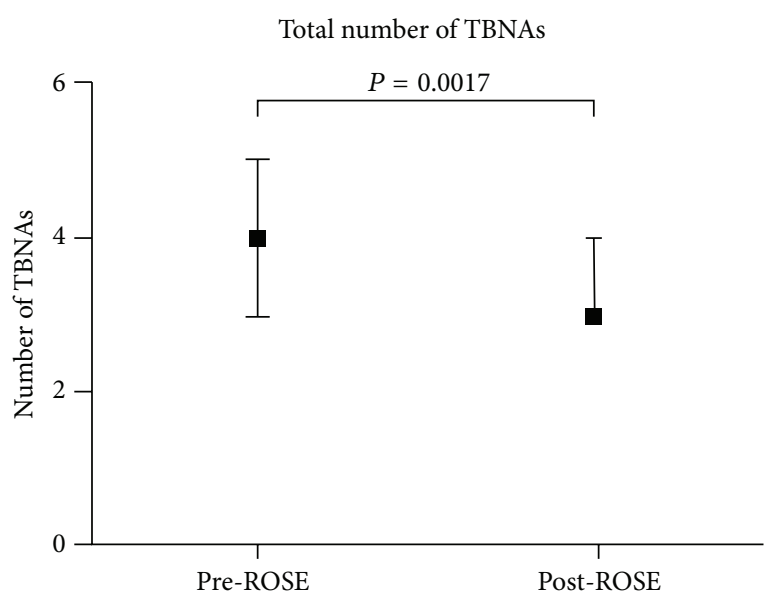

FIgURe 1: Data comparison graph.

metastatic colorectal cancer, poorly differentiated, malignant, diffuse large B cell lymphoma, and nocardia infection. Fifty had normal lymphocytes with no pathology.

Mean number of lesions sampled was significantly fewer in the post-ROSE group than in the pre-ROSE group (mean: $1.5 \pm 0.7(1,4)$ versus $1.9 \pm 0.8(1,4), P=0.0020)$. In the post-ROSE group, the mean number of punctures was lower than in the pre-ROSE group (mean: $3.6 \pm 1.4(1,8)$ versus $4.2 \pm 1.5(1,8), P=0.0017)$. This is displayed in Figure 1. ROSE also resulted in a reduction in the number of lesions biopsied ( $45 \%$ of $>1$ lesion biopsied versus $66 \%$ in the preROSE group). The mean number of TBNAs per lesion was not significantly different between the two groups. The summary of findings is listed in Table 2.

With regards to the diagnostic performance for suspected lung cancer, the concordance rate between ROSE and final cytological diagnosis was $94 \%$. This is summarised in Table 3. ROSE detected malignancy in 51 cases, 26 granulomas with the remaining 31 being nondiagnostic.
TABLE 2: Summary of findings.

\begin{tabular}{lccc}
\hline & Pre-ROSE & Post-ROSE & $P$ value \\
\hline Number of cases & 66 & 112 & - \\
Mean number of lesions & $1.9 \pm 0.8$ & $1.5 \pm 0.7$ & 0.0020 \\
& $(1,4)$ & $(1,4)$ & \\
Mean number of TBNAs & $\begin{array}{c}4.2 \pm 1.5 \\
(1,8)\end{array}$ & $\begin{array}{c}3.6 \pm 1.4 \\
(1,8)\end{array}$ & 0.0017 \\
Mean number of TBNAs & $2.5 \pm 1.0$ & $2.5 \pm 0.9$ & 0.35 \\
per lesion & $(0.7,5)$ & $(1,5)$ & \\
$>1$ lesion investigated & 66 & 45 & - \\
$(\%)$ & &
\end{tabular}

Results expressed as mean \pm standard deviation (range).

TABLE 3: ROSE and correlation to final cytology results.

\begin{tabular}{lcc}
\hline \multirow{2}{*}{ ROSE } & \multicolumn{2}{c}{ Final cytology } \\
& Malignant & Nonmalignant \\
\hline Positive for malignancy & 51 & 0 \\
Negative for malignancy & 3 & 58 \\
\hline Total & 54 & 58 \\
\hline
\end{tabular}

Sensitivity: $51 / 54 \times 100=94.44 \%$.

Specificity: $58 / 58 \times 100=100 \%$.

Positive predictive value (PPV): $51 / 51 \times 100=100 \%$.

Negative predictive value $(\mathrm{NPV}): 58 / 61 \times 100=95 \%$.

The results of ROSE correlated well with the final cytologic diagnosis, which was considered the gold standard for positive results, while an alternative nonmalignant diagnosis or resolution on follow-up was considered the gold standard for negative results. There were three cases of false negatives with ROSE. In one of the false negative cases, the first two passes were negative for malignancy; however, the subsequent two passes were not screened by ROSE as the procedure was aborted due to poor patient tolerance. The final diagnosis was confirmed from the cell block to be large B cell nonHodgkin lymphoma. The other two false negative cases were reported as small cell carcinoma in final cytology. These findings are listed in Table 4.

The sensitivity and specificity of ROSE compared to final cytology results were $94.4 \%$ and $100 \%$, respectively. ROSE had a positive predictive value of $100 \%$ and a negative predictive value of $95 \%$.

The pathology from 163 cases (53 non-ROSE and 110 ROSE) was available for review by one of the authors (Kerryn Ireland-Jenkin). For the purpose of this study, cell blocks were classified as satisfactory if they contained either the same diagnostic material as the conventional cytology smears or better. It was important to include data on cell block rates due to the potential benefit of being able to perform additional pathology tests, including immunohistochemistry and molecular pathology on the sample.

Pre-ROSE, 74\% of EBUS cases yielded a satisfactory cell block and when ROSE was performed, $90 \%$ of cases generated at least one satisfactory cell block. ROSE had the additional benefit of a cytologist controlling the cytology specimen, and in this setting, there were no cases where diagnostic material 
TABLE 4: False negative cases.

\begin{tabular}{|c|c|c|}
\hline Target/s & ROSE result & Final cytology results \\
\hline $\begin{array}{l}\text { LN 7, } \\
\text { nodule }\end{array}$ & $\begin{array}{l}\text { Lymphocytes only with } \\
\text { possible enlarged } \\
\text { lymphocyte in LN } 7\end{array}$ & $\begin{array}{l}\text { Small cell carcinoma. } \\
\text { Immunohistochemistry } \\
\text { positive for synaptophysin, } \\
\text { chromogranin, and CD } 56 .\end{array}$ \\
\hline $3 p$ & $\begin{array}{l}\text { Lymphocyte and bronchial } \\
\text { cells in 1st and } 2 \text { nd passes. } \\
3 \text { rd and } 4 \text { th passes not } \\
\text { viewed by ROSE. }\end{array}$ & $\begin{array}{l}\text { Poorly differentiated cancer } \\
\text { in 3rd and 4th passes. } \\
\text { Immunohistochemistry } \\
\text { revealed large B cell } \\
\text { non-Hodgkin lymphoma. }\end{array}$ \\
\hline $\begin{array}{l}11 \mathrm{~L}, 4 \mathrm{~L}, \\
11 \mathrm{R}\end{array}$ & Lymphocytes & Malignant in $11 \mathrm{R}$ \\
\hline
\end{tabular}

TABLE 5

\begin{tabular}{lcc}
\hline & Non-ROSE & ROSE \\
\hline Total cases & 66 & 112 \\
Number reviewed & 53 & 110 \\
Number with cell block histology & $48(91 \%)$ & $105(95 \%)$ \\
No cell block attempted & $5(9 \%)$ & $5(5 \%)$ \\
$\begin{array}{l}\text { Number with satisfactory cell block } \\
\text { Number with no (or poor quality) }\end{array}$ & $39(74 \%)$ & $99(90 \%)$ \\
cell block & $14(26 \%)$ & $11(10 \%)$ \\
\hline
\end{tabular}

was compromised by making a cell block. The summary of findings is listed in Table 5.

The higher rate of satisfactory cell-block creation was due to ROSE confirming specimen adequacy, therefore improving the amount of quality material available for testing and the additional contribution made by the cytologist controlling the distribution of the material. In this study, the creation of a satisfactory cell block was regarded as an important pathology outcome, as it is crucial to further testing (such as immunohistochemistry, molecular testing, or mycobacterial PCR) on a cytology sample. For the purpose of this study a cell block was deemed adequate if it contained the same (or more significant) diagnostic material as the smeared slides.

The creation of a diagnostic cell block will reduce the rate that the final diagnosis will be non-small cell lung cancer (NSCLC) not otherwise specified (NOS) by definition, since the specific diagnosis of squamous or glandular differentiation in lung carcinoma currently relied heavily on tumour immunophenotype. In addition to this, the reasons for choosing creation of a satisfactory cell block (with diagnostic material) as the pathology end-point included the objective nature of this assessment and its applicability equally to malignant and nonmalignant cases irrespective of the disease process. For a variety of reasons, immunohistochemistry was not performed in all cases where malignancy was diagnosed and confirmed in the cell block. Also, in some instances, cell block immunohistochemistry provided useful information other than lung carcinoma immunophenotype (such as confirming metastatic tumour, NHL, or the presence of mediastinal thyroid tissue). Therefore, if lung carcinoma phenotype alone had been used as the pathology end-point, the results would have been less representative of the true value of ROSE.
The only complications observed in our study were excessive coughing and irritability after procedure. There were no serious or life-threatening complications.

\section{Discussion}

This study demonstrated that during EBUS-TBNA, ROSE led to a significant reduction in mean number of lesions sampled and a reduction in mean number of lymph node TBNAs performed. Despite the reduction in the number of lesions sampled, the use of ROSE also led to an increase in proportion of cases where an adequate cell block was prepared. ROSE allowed the bronchoscopist to alter the way in which tissue sampling was performed to optimize tissue acquisition with minimal number of lesions targeted.

The utility of ROSE during EBUS-TBNA has been reported in literature. There had been conflicting results up to date. However, a high concordance rate is reported in a recent study [11]. In our study, we found that for suspected lung cancer cases, the concordance rate between ROSE and final cytologic diagnosis was $94 \%$. This good rate was thought to be due to the experience of the team of pathologists and bronchoscopists in our institution.

The presence of ROSE during EBUS-TBNA alerted the bronchoscopist to the adequacy of samples, as well as determining the need for additional sampling for ancillary immunohistochemical and molecular testing. This is of great importance in this era of personalized medicine in the management of lung cancer. We believe that the use of ROSE during EBUS-TBNA was beneficial to facilitate these necessary ancillary tests.

We found that the use of ROSE was associated with a reduction in the number of lesions sampled (66\% patients in pre-ROSE with $>1$ lesion investigated compared with $45 \%$ in post-ROSE). This was because the procedure could be terminated after adequate tissue was obtained rather than sampling all pathologic lymph nodes. This was important as sampling of multiple lymph node stations may have increased the duration of bronchoscopy and may lead to higher complication rates.

The sensitivity and specificity of ROSE were high in our study which was similar to those observed in other studies [12]. The presence of no false positive suggested that ROSE during EBUS-TBNA was confident in detecting a malignant diagnosis.

One limitation of this study was the retrospective study design. The impact of this was minimised by including all patients who had had EBUS-TBNA leading to a larger sample size. A randomised prospective trial evaluating the utility of ROSE would help strengthen our conclusions and support for ROSE. Another limitation was that this study was performed in a single institution. Therefore, generalisation to other institutions may be limited.

\section{Conclusion}

In conclusion, ROSE during EBUS-TBNA contributed to a significant reduction in the number of TBNA samples 
performed per case and a greater number of cases where an adequate cell block was prepared. This has important implications for immunohistochemical and molecular testing to facilitate management of lung cancer. ROSE was more beneficial to off-site cytological assessment of bronchoscopy specimens. Off-site assessment of TBNA samples during linear EBUS was an inadequate substitute for ROSE.

\section{Disclosure}

No portion of this work has been published previously or is under consideration for publication elsewhere and this is an original paper. The authors declare no financial support for this work.

\section{Conflict of Interests}

All authors do not have potential conflict of interests.

\section{References}

[1] F. Herth, H. D. Becker, and A. Ernst, "Conventional vs endobronchial ultrasound-guided transbronchial needle aspiration," Chest, vol. 125, no. 1, pp. 322-325, 2004.

[2] K. Yasufuku, M. Chiyo, Y. Sekine et al., "Real-time endobronchial ultrasound-guided transbronchial needle aspiration of mediastinal and hilar lymph nodes," Chest, vol. 126, no. 1, pp. 122-128, 2004.

[3] K. Yasufuku, T. Nakajima, K. Motoori et al., "Comparison of endobronchial ultrasound, positron emission tomography, and CT for lymph node staging of lung cancer," Chest, vol. 130, no. 3, pp. 710-718, 2006.

[4] D. Baram, R. B. Garcia, and P. S. Richman, "Impact of rapid onsite cytologic evaluation during transbronchial needle aspiration," Chest, vol. 128, no. 2, pp. 869-875, 2005.

[5] R. Chin Jr., T. W. McCain, M. A. Lucia et al., "Transbronchial needle aspiration in diagnosing and staging lung cancer: how many aspirates are needed?" American Journal of Respiratory and Critical Care Medicine, vol. 166, no. 3, pp. 377-381, 2002.

[6] A. H. Diacon, M. M. Schuurmans, J. Theron et al., "Utility of rapid on-site evaluation of transbronchial needle aspirates," Respiration, vol. 72, no. 2, pp. 182-188, 2005.

[7] G. B. Diette, P. White Jr., P. Terry, M. Jenckes, D. Rosenthal, and H. R. Rubin, "Utility of on-site cytopathology assessment for bronchoscopic evaluation of lung masses and adenopathy," Chest, vol. 117, no. 4, pp. 1186-1190, 2000.

[8] R. D. Davenport, "Rapid on-site evaluation of transbronchial aspirates," Chest, vol. 98, no. 1, pp. 59-61, 1990.

[9] F. J. F. Herth, R. Eberhardt, P. Vilmann, M. Krasnik, and A. Ernst, "Real-time endobronchial ultrasound guided transbronchial needle aspiration for sampling mediastinal lymph nodes," Thorax, vol. 61, no. 9, pp. 795-798, 2006.

[10] F. J. F. Herth, M. Krasnik, K. Yasufuku, R. Rintoul, and A. Ernst, "Endobronchial ultrasound-guided transbronchial needle aspiration," Journal of Bronchology, vol. 13, no. 2, pp. 84-91, 2006.

[11] T. Nakajima, K. Yasufuku, F. Saegusa et al., "Rapid on-site cytologic evaluation during endobronchial ultrasound-guided transbronchial needle aspiration for nodal staging in patients with lung cancer," Annals of Thoracic Surgery, vol. 95, no. 5, pp. 1695-1699, 2013.
[12] M. Oki, H. Saka, C. Kitagawa et al., "Rapid on-site cytologic evaluation during endobronchial ultrasound-guided transbronchial needle aspiration for diagnosing lung cancer: a randomized study," Respiration, vol. 85, no. 6, pp. 486-492, 2013. 


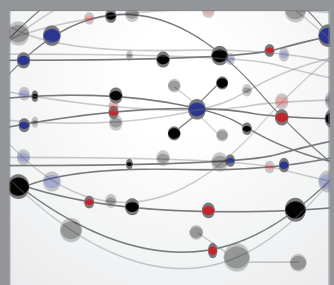

The Scientific World Journal
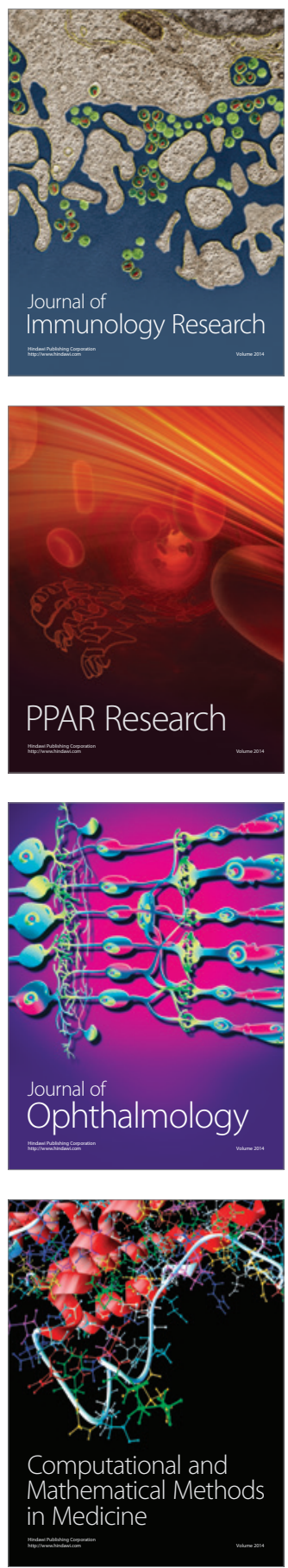

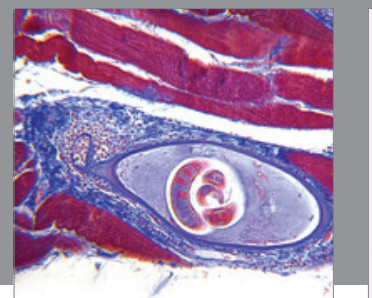

Gastroenterology

Research and Practice
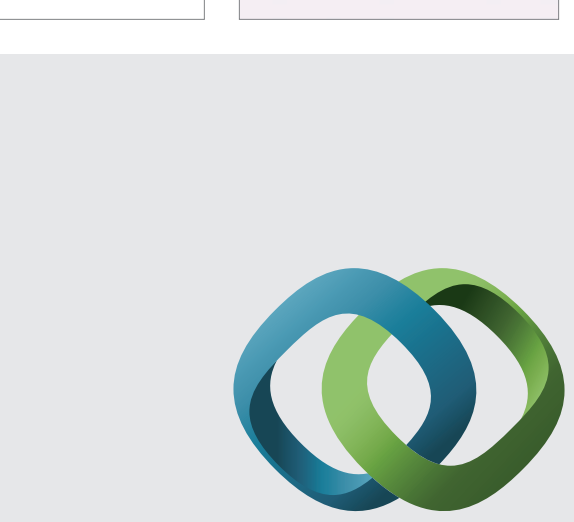

\section{Hindawi}

Submit your manuscripts at

http://www.hindawi.com
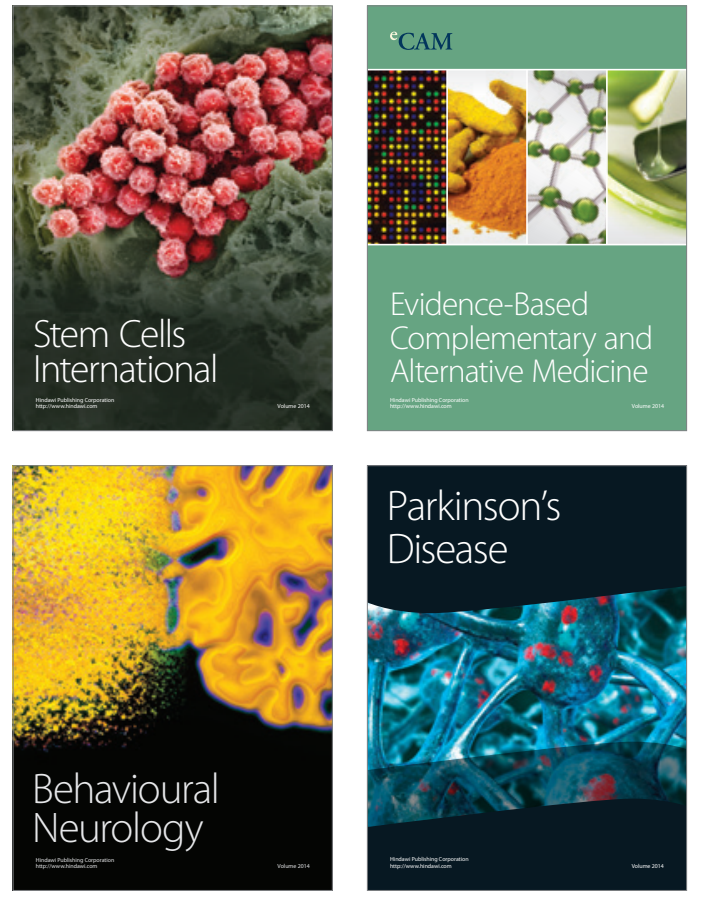
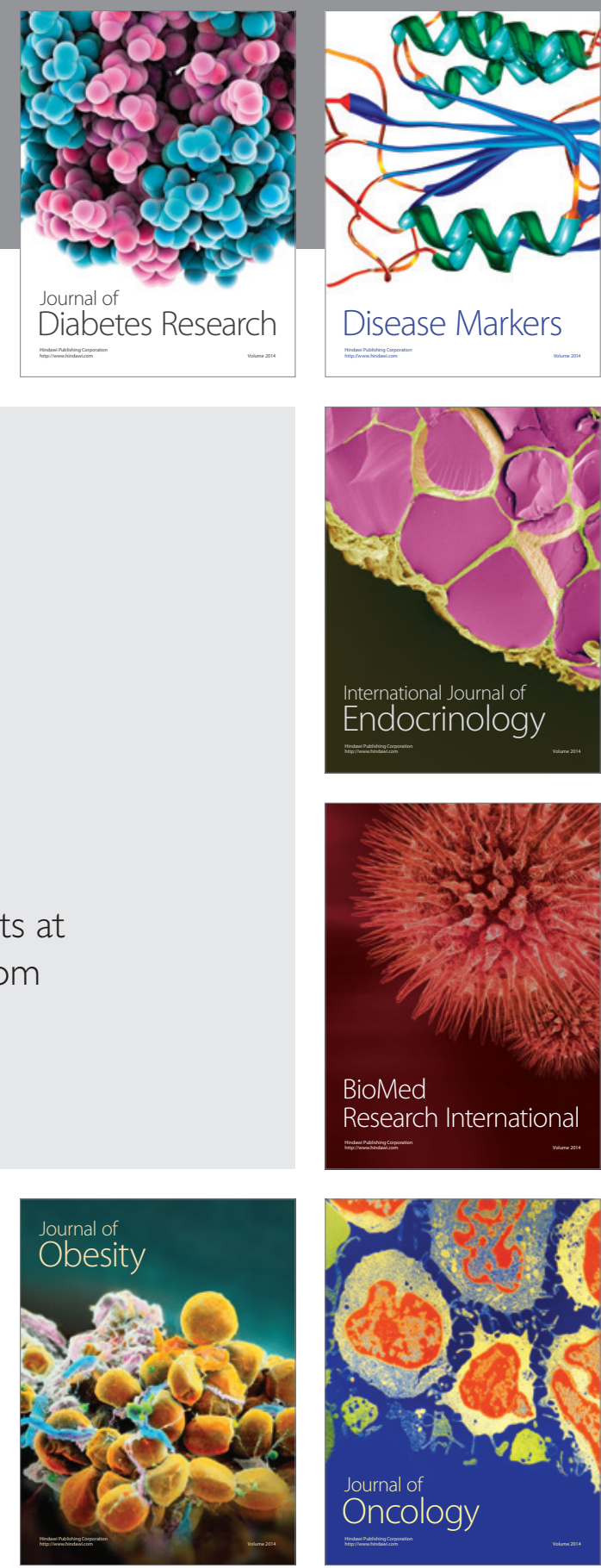

Disease Markers
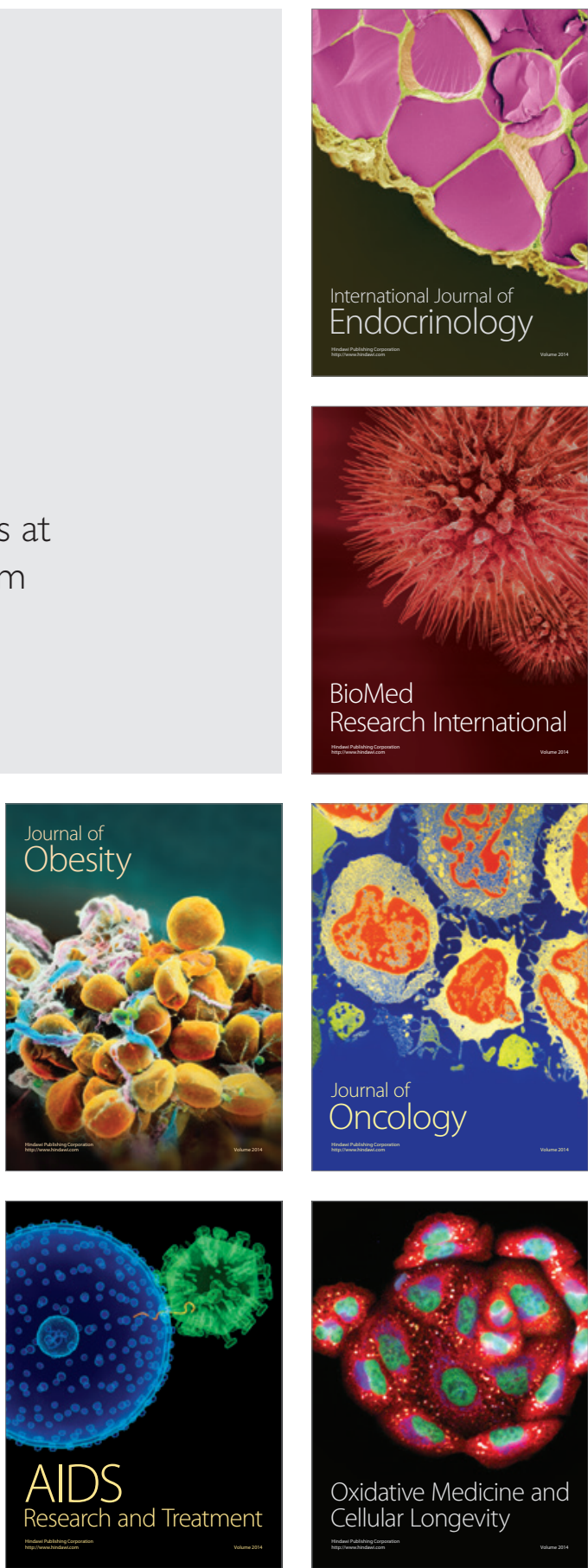